\title{
Estimated workload intensity during volunteer aquarium dives
}

by

Buzzacott $\mathrm{P},{ }^{1,2^{*}}$ Grier JW, ${ }^{3}$ Walker J, ${ }^{4}$ Bennett CM, ${ }^{1}$ Denoble PJ. ${ }^{1}$

${ }^{1}$ Divers Alert Network, Durham, North Carolina, USA

${ }^{2}$ School of Sports Science Exercise and Health, University of Western Australia, Crawley, Western Australia, Australia

${ }^{3}$ Department of Biological Sciences, North Dakota State University, Fargo, North Dakota, USA.

${ }^{4}$ Oregon Coast Aquarium, Newport, Oregon, USA

*Corresponding author

\section{Abstract}

Background: This study aimed to characterise the physiological demands of working dives on volunteer divers at a public aquarium in the US.

Aims: To estimate the workloads associated with volunteer dives in a US aquarium.

Methods: Participants completed a medical and diving history questionnaire. Measurements included blood pressure before and after diving and continuous ECG (Holter) monitoring during diving. Dive profiles were recorded using loggers. Mean workload was estimated from total air consumption.

Results: Twenty-seven divers recorded 49 air dives over five days. Two-thirds were male and ages ranged from 40-78 years. Typically, each diver made two dives with a 30-60 minutes surface interval. Mean heart rate while diving was 100 beats per minute. Mean estimated workload during the dives recorded during this study was 5.8 METS, with a range from 4.1 to 10.5. The highest mean recorded heart-rate was $120 \mathrm{bpm}$ over 40 minutes, vacuuming the floor in the shark exhibit.

Conclusions: Given the mean age of this sample and the prevalence of cardiovascular risk factors (BMI, high cholesterol, and hypertension), it may be prudent for aquariums to regularly monitor $\mathrm{SAC} / \mathrm{kg}$ and heart rate in volunteer divers, to identify which tasks require the highest workload intensity. Divers with existing cardiovascular risk factors might then be employed in dives with lighter workloads. In conclusion, volunteer dives at this aquarium required a mean workload intensity that was described by recreational divers as moderate. The highest workload, at 10 METS for 23 minutes, would be considered by many recreational divers as exhausting. 


\section{Estimated workload intensity during volunteer aquarium dives}

\section{Introduction}

There are at least 80 public aquariums in the USA.(1) Many employ volunteer divers to clean the exhibits, including vacuuming the bottom and/or cleaning acrylic windows from the inside. It is not currently known what level of workload these activities require. Immersion itself imposes stress on the cardiovascular system due to blood shift from the periphery to the chest cavity.(2) This results in increased stroke volume of the heart and decreased heart rate. Underwater work increases demand for oxygen and, consequently, both stroke volume and heart rate increase which requires a healthy cardiovascular system able to adapt. On the other hand, aquarium volunteer divers are often seniors,(3) and the ability of their cardiorespiratory system to meet increased oxygen demands while diving is not known.

When a diver descends underwater the ambient pressure increases substantially. By a depth of $10 \mathrm{~m}$ in the sea a diver is at two atmospheres of pressure. The Self-Contained Underwater Breathing Apparatus (scuba) delivers breathing gas, usually air, at ambient pressure. This denser gas diffuses into the bloodstream for distribution around the body. The process is reversed when a diver returns to the surface. Potential hazards associated with scuba diving include drowning, hypothermia, and acute coronary events.

Oxygen consumption $\left(\mathrm{VO}_{2}\right)$ of working divers was estimated previously by measuring scuba gas tank pressure before and after diving.(4) Workload while diving has been estimated in METS, based on minute ventilation $\left(\dot{V}_{E}\right),(5)$ and by measured exhaled gas.(6-8) This latter method has also been used in conjunction with heart-rate. The correlation between $\mathrm{VO}_{2}$ or $\dot{V}_{E}$ and heart rate has been measured before,(9-11) but not in aquarium divers. Knowledge of workload intensity and heart-rate is important because diving safety officers can then direct volunteer divers to easier or more strenuous tasks according to their history of cardiovascular risk factors. This study aimed to characterise the physiological demands of working dives conducted at a public aquarium by volunteer divers.

\section{Methods}

In October 2017, volunteer divers at a US regional aquarium of approximately 3.5ML were invited to participate. The aquarium has three exhibits; one large open sea exhibit containing many sharks (OS), one exhibit representing the inshore environment (IS) and one representing a nearby reef ecosystem (RE). In the OS tasks include suction vacuuming the gravel on the floor and cleaning the Perspex walkway that runs through the exhibit. In the IS the surface area of both the viewing windows and the floor are smaller than in the OS, and the RE is the smallest of the three exhibits.

As volunteer divers arrived in the morning, each gave signed informed consent and a completed medical and diving history questionnaire. They had blood pressure measured in both arms using an electronic blood pressure monitor (Model BP761N, Omron Healthcare Co 
Ltd, Muko, Kyoto, Japan). Ultrasound images of each diver's heart were recorded for a separate, concurrent study. Most of the divers were then connected to a customized-encased waterproof 3-channel Holter ECG recorder (model H3+, H-Scribe v4.34 software, Mortara Instrument, Milwaukee, WI). All divers were next connected (simultaneously for those also connected to the Holter) to a 12-lead ECG system (IQmark, Midmark International, Dayton, $\mathrm{OH})$ and rested supine approximately 15 minutes for resting heart-rate and other variables not reported in this study. Finally, the 12-lead ECG was disconnected, the electrode patches left in situ, Holter recorders were left recording on the diver, and each diver was given a Sensus Ultra logger (ReefNet, Mississauga, Canada) with instructions to attach it to the front of the Buoyancy Control Device (BCD). The Sensus loggers employed a sampling rate of one record every ten seconds.

The divers then walked approximately $100 \mathrm{~m}$ to the aquarium where they dressed for diving, most commonly in drysuits. Only divers wearing drysuits wore a Holter ECG while diving.

Each dive was logged by a Dive Safety Officer (DSO) at the surface, including the date, tank size, start/end times, start/end air pressures (from each diver's submersible pressure gauge), which exhibit each diver dived in and the task performed. Recorded differences in pre-post dive tank pressures were converted from PSI to bar by multiplying with a factor of 0.069 . Typically each diver made two dives with a 30-60 minutes surface interval between them. All dives were made breathing compressed air. After each diver completed their last dive for this study, the divers walked back to the research area where they had blood pressure again measured in each arm, a second ultrasound recording of their heart, the 3-lead ECG Holter recorder was left attached, and rested supine for another 15 minutes for post-dive ECG measurements. Meanwhile, the returned Sensus loggers were downloaded using Sensus Manager (ReefNet, Mississauga, Canada). Depths and water temperature were sampled through each dive every ten seconds. The ECG recordings were analysed using IQmanager Diagnostic Wokstation v8.4.1 (Midmark International, Dayton, $\mathrm{OH}$ ). No diver participated on more than one day. The study protocol was approved by the Institutional Review Board of Divers Alert Network, approval number 015-17.

For analysis, the beginning of each dive was defined as when the loggers first descended below $1.0 \mathrm{~m}$ depth and the end of each dive when they last ascended above $1.0 \mathrm{~m}$ depth. These were rounded up to whole minutes and start-to-end times were noted. The mean depth of each dive was calculated by dividing the sum of all depths recorded between the start and end of the dive by the number of samples collected.

The mean heart-rate over the duration of each dive was calculated from the Holter ECG recordings in a multi-stage process that converted the data to more portable and standard sampling (ms) and file (csv) formats. The conversion permitted more flexibility for data analyses, including correction for artefacts using the "very strong" artefact correction setting of Kubios HRV Premium v.3.0.0 software (Kubios Ltd., Kuopio, Finland).

The total volume of breathing gas consumed was calculated from the manufacturer's specified internal volumes for each tank used, multiplied by recorded pressure drop. Each 
diver's mean Surface Air Consumption (SAC) rate was estimated. This is equivalent to the mean volume of gas the diver would have breathed at the surface during one minute $\left(\dot{V}_{E}\right)$ for the same mean level of exercise. These SAC were divided by the divers' self-reported body weight to yield $\mathrm{SAC} / \mathrm{kg}$ for each diver during each dive. These were converted to dimensionless metabolic equivalents (METS) using the method previously described,(5) with resting metabolic rates of oxygen consumption of $3.5 \mathrm{~mL} \cdot \mathrm{kg}^{-1} \cdot \mathrm{min}^{-1}$ for males and $3.2 \mathrm{~mL} . \mathrm{kg}^{-}$ ${ }^{1} . \mathrm{min}^{-1}$ for females, as recommended by Pollock.(12)

The oxygen consumption rate per minute $\left(\dot{V} O_{2}\right)$ was estimated indirectly based on previously established relationship between $\dot{V} O_{2}$ and minute pulmonary ventilation $\left(\dot{V}_{E}\right){ }^{5}$

$$
\text { (1) } \dot{V} O_{2}=0.0256 \dot{V}_{E}+1.070
$$

Demographic summary statistics and correlation coefficients between SAC/kg or METS and mean heart rate were calculated using SAS ver 9.4 (SAS, Cary, NC). The batteries of three Sensus loggers expired during the study and five recorded dive profiles were irretrievably lost. Start times, end times and mean depths were compared between 24 buddy pairs. Correlation coefficients $(r)$ were 1.0, 0.92 and 0.93 respectively and so the start times, end times and mean depths of the dive buddy were substituted for the five dives lost due to battery failure.

\section{Results}

Anthropometry of the divers are presented in Table 1. Two thirds were male and ages ranged from 40-78 years. Nine of the 27 divers (33\%) reported taking prescription medication for cholesterol, 10 (37\%) reported taking prescription medication for hypertension and 17 (63\%) overall reported taking at least one prescription medication.

Twenty-seven divers recorded 49 dives over five days. The diver with the longest diving history was first certified to dive in 1970, the newest in 2015. Mean heart rate was recorded for 30 dives, the Holter ECG recorder was worn but the signal was not recorded on 7 dives and no Holter recorder was worn during 12 dives due to there being more divers than recording units at some times.

There were no significant differences between pre- and post- dive resting blood pressures. Dive characteristics are described for dives in each exhibit respectively, in Table 2.

Maximum ascent rate of any dive was $17 \mathrm{~m} \cdot \mathrm{min}^{-1}$ while mean maximum ascent rate overall was $9.8 \mathrm{~m} \cdot \mathrm{min}^{-1}$. Overall, the mean estimated workload during the dives recorded by this study was 5.8 METS, with a range from 4.1 to 10.5 . The highest mean recorded heart-rate was 120 bpm over 40 minutes, while vacuuming the floor in the shark exhibit.

The relationship between mean heart rate and SAC. $\mathrm{kg}^{-1}$ had a correlation coefficient $(r)$ of 0.62 , and the correlation between heart rate and estimated $\dot{V} O_{2}$ was 0.43 . 
A comment was made by one of the returning divers that he perceived diving in the OS exhibit to be more demanding than the other exhibits because the divers were constantly looking around for approaching sharks. It has previously been reported that shark swimming patterns become unpredictable when divers enter this particular shark exhibit.(13) During his dive in the OS a large shark had approached the buddy pair and they reported feeling as though their heart rates had momentarily increased in response. On later examination, both Holter recordings showed similar, simultaneous brief spikes in heart rate to over $140 \mathrm{bpm}$. To further investigate the hypothesis of increased demand in the OS exhibit, a nested sub-set of matched dives, made by divers who dived in the OS and one other exhibit, was created. Seventeen divers recorded dives in both the OS exhibit and one of the other two. Details are presented in Table 3.

\section{Discussion}

Overall the mean estimated workload during these aquarium dives was 5.8 METS, which is a level reported by recreational divers as a moderate workload.(5) The highest estimated workload recorded, while cleaning the acrylic windows in the shark exhibit, was 10.5 METS which is above the level recreational divers have described as severe or exhausting.(5) Mean heart rate during this 23 minutes dive was $119 \mathrm{bpm}$.

It should be remembered, however, that the estimated workload intensities reported in this study are based on tank pressure drop between the start and end of the dive, and those pressure differentials may have been exaggerated through tank cooling, inflation of the BCDs and drysuits, mask clearing, equalisation and/or gas lost during free-flow events. Therefore, the values presented in this report should be viewed as maximums, with actual consumption rates and workloads likely less. An adjustment due to an arbitrary 10\% assumed gas loss was applied in a previous study,(5) but was not applied in this study. The former study had maximum depths that were three times greater than in this study, with recreational divers in the sea frequently changing depths and adjusting the volume of their buoyancy compensators, whereas in this study participants would reach the depth required for a task and there was less need to adjust the volume of gas in the BCD.

That no difference was detected between pre- and post- dive blood pressure may have been influenced by the delay between surfacing and the post-dive recording, since the divers were required to remove and rinse their dive equipment, then walk approximately $100 \mathrm{~m}$ to the data collection station. The correlation between heart rate and estimated $\dot{V} O_{2}$ was 0.43 , which is much lower than $r=0.74$ found among younger (mean age 26 years) male divers exercising heavily underwater in wetsuits, with an ergometer, at depth of 10msw.(6) The difference could be due to numerous factors including not only age, workload, water temperatures and protective equipment (including weighting), and depths, but also differences in other equipment, individual divers, diving techniques, variability in activity and nature of the dives, and such things as brief spikes in heart rates that might affect mean HR more than mean SAC. The difference also calls into question the validity of adjusting decompression 
obligations for recreational divers based on heart-rate inferred workload, as some modern dive computers claim to do. Heart rate in divers is highly variable and influenced by factors other than mere workload, for example immersion, water temperature, exposure protection, gas density and many others. Heart rate was also found to be a poor predictor of $\mathrm{VO}_{2}$ and, by definition, of workload intensity in helium-oxygen saturation dives.(14)

Given the mean and range of diver ages in our sample and the prevalence of cardiovascular risk factors (BMI, high cholesterol, and hypertension) in some, it may be prudent for aquarium DSOs to regularly monitor $\mathrm{SAC} / \mathrm{kg}$ and heart rate in volunteer divers, to identify which tasks consistently require the highest workload intensity, so those divers with existing cardiovascular risk factors might be employed in dives with lighter workloads. While this study identified which exhibit involved tasks with, on average, higher estimated workloads, further research should investigate specific tasks within each exhibit, e.g. vacuuming vs. cleaning windows.

Despite reported feelings of a higher workload, dives in the shark exhibit showed no significant differences compared to dives made by the same divers in other exhibits, in mean heart rate, air consumption, estimated oxygen consumption or workload (METS). Any anxiety or stress responses associated with diving in the shark exhibit did not manifest as an overall greater cardiopulmonary response. A limitation of this study design was that the divers always dived in the RE or IS exhibits first, before making any dives in the relatively warmer shark exhibit. However, in this study there was no detectable association between heart rate and water temperature (Table 3 ).

In conclusion, volunteer dives at this aquarium required a mean workload intensity that equated to that described by recreational divers as moderate. The highest workload, at 10 METS for 23 minutes, would be considered by many recreational divers as exhausting.

\section{Acknowledgements}

Thanks are given to the management and volunteer divers at the Oregon Coast Aquarium. This study was entirely funded by Divers Alert Network.

\section{Legend of Tables and Figures}

Table 1: Anthropometry and dive characteristics of volunteer aquarium divers ( $n=27$ )

Table 2: Dive characteristics and physiological variables during volunteer aquarium dives $(n=49)$

Table 3: Dive characteristics and physiological variables during volunteer aquarium dives in the shark exhibit ( $n=17)$ or the other exhibits $(n=17)$ matched by the same divers

\section{References}


1. Aquariums and Marine Life Centers of the World [Internet]. MarineBio Conservation Society; 2018 [cited 2018 Feb 14th]. Available from: http://marinebio.org/marine-aquariums/.

2. Åsmul K, Irgens Å, Grønning M, Møllerløkken A. Diving and long-term cardiovascular health. Occup Med (Lond) 2017;67:371-6.

3. Smith C, Buckley N, Bridges E, Pavitt B, Moss A. Self-reported impacts of volunteering in UK zoos and aquariums. Cultural Trends 2018;27(1):18-32.

4. Lanphier EH. Oxygen consumption in underwater swimming. Fed Proc 1954;13:84.

5. Buzzacott $P$, Pollock NW, Rosenberg $M$. Exercise intensity inferred from air consumption during recreational scuba diving. Diving Hyperb Med 2014;44:74-8.

6. Dwyer J. Estimation of oxygen uptake from heart rate response to undersea work. Undersea Biomed Res 1983;10:77-87.

7. Pendergast DR, Tedesco M, Nawrocki DM, Fisher NM. Energetics of underwater swimming with SCUBA. Med Sci Sports Exerc 1996;28:573-80.

8. Jones RL, Docherty D, Gaul CA, Goulet LL, McFadyen PF, Hartley TC, et al. Ventilatory gas analysis in SCUBA divers using a surface-based measurement system. Undersea Hyperb Med 2007;34:341-8.

9. Dwyer J, Saltzman HA, O'Bryan R. Maximal physical-work capacity of man at 43.4 ATA. Undersea Biomed Res 1977;4:359-72.

10. Craig AB, Dvorak M. Comparison of exercise in air and in water of different temperatures. Med Sci Sports 1969;1(3):124-30.

11. Spaur WH, Raymond LW, Knott MM, Crothers JC, Braithewaite WR, Thalmann ED, et al. Dyspnea in divers at 49.5 ATA: mechanical not chemical in origin. Undersea Biomed Res 1977;4:18398.

12. Pollock NW. Aerobic fitness and underwater diving. Diving Hyperb Med 2007;37:118-24.

13. Hodges V, Frierson TN. Diving with captive sharks. In: Brueggeman P, Pollock NW. Diving for Science 2008; Dauphin Island, AL: American Academy of Underwater Sciences; 2008. p. 53-70.

14. Shykoff $\mathrm{BE}$, Knafelc ME. Exercise heart rate as a predictor of oxygen consumption during decompression from saturation diving. Panama City, FL: Navy Experimental Diving Unit, 2002. 